I venture to briefly recapitulate the therapeutic propositions which I have advanced:-

1st. For the secondary forms of syphilis give, in the most eligible form, iodide of potassium in moderate doses, with blue pill or some analogous preparation.

2nd. The double compound of iodide of potassium and bichloride of mercury may be necessary for treating syphilitic inflammation of deeper tissues.

3rd. The bichloride of mercury is valuable for removing the intermediary squamous syphilides. The green iodide of mercury is recommended by Mr. Squire. In doubtful cases, bichloride of mercury may be added to arsenic.

4th. The earlier tertiary phenomena of syphilis are relieved by the double compound of iodine and mercury just named; the later ones by large doses of iodide of potassium. Sometimes even cures may be effected. Tonics are generally useful subordinate agents.

5th. Infantile syphilis always requires mexcury.

And I may add that in otherwise intractable cases, the mercurial bath and the hypodermic method of introducing mercury into the system should be rendered available.

Bath, June, 1869.

\section{PRIMARY AMPUTATION OF BOTH LOWER LIMBS. \\ RECOVERY.}

By JAMES CARMTCHAEL, M.D.

IN primary amputations the mortality is always great. When we take into account the extent of the injury, along with the sudden and great shock to the system, and the delay which in many cases must occur before medical assistance can be procured, this result is scarcely to be wondered at. In the following case the patient lost both lower limbs from an accident on a railway. Recovery under these circumstances is so unusual that I have felt it a duty to record the case.

A. G-, aged forty, while loading his cart with coals from a waggon at a siding on the railway, was suddenly knocked down by the truck being unexpectedly put in motion by an engine, and some other trucks coming up against it. He most unfortunately fell before the waggon, which went over both his legs. On seeing the patient, shortly after, I found him much collapsed, but not losing blood. Stimulants were administered, and he was removed as speedily as possible to his own house, not far distant. On examination, it was found that the left leg was almost completely severed just below the knee; the right was much mangled in its lower third, the bones being very much comminuted, the vessels and muscles torn and crushed, and the skin stripped up from the subjacent textures nearly as far as the tibial tuberosity in front. The patient having slightly rallied, it was decided to remove the limbs at once.

With the help of my colleague, Dr. Williamson, who throughout kept the patient carefully under the infuence of chloroform, and a non-professional assistant, I proceeded to the operation, first upon the right limb, which was removed below the knee by making two semilunar flaps of equal dimensions, the one on the antero-internal, the other on the postero-external aspect of the limb. The skin in both cases being dissected freely up, the muscles were divided, and the bone sawn through just below the tuberosity of the tibia; care being taken to round off the anterior edge of the bone by entering the saw at first obliquely, and having withdrawn it, applying it again a little below. One vessel was ligatured, and the rest secured by torsion. The flaps having been brought together by a few interrupted metallic sutures, the stump was dressed with lint soaked in carbolic oil. At this period of the operation the patient became very low, and it became necessary to remove the other limb with all possible speed. This was done with great ease and rapidity by a long anterior and short posterior flap. The skin of the anterior flap having been dissected up, the muscles were divided obliquely up to the point where the bone was to be sawn; posteriorly a short flap was made in a similar manner. Hardly any bleeding occurred; a liga- ture was, however, put on the femoral, and several smaller | arteries were twisted. The stump was dressed in a similar manner to its fellow.

The patient was put to bed, and with the aid of hot bottles, extra blankets, and stimulants, he gradually began to rally, although for some short time after the operation he appeared to be sinking.

The operation was performed at 1 P.Mr.

In the evening, the patient seems to have recovered al. most entirely from the immediate effects of the accident and operation. Countenance placid and natural; expresses himself as pretty well. Pulse 88, of good strength. Ordered beef-tea, and less of the brandy, and forty-five minims of solution of muriate of morphia.

Jan. 14th.-Reaction. Skin warm; face flushed; complains of slight headache and thirst. Pulse 104. Ordered to omit stimulants, continue beef-tea, and milk-and-water ad libitum to drink. Serous discharge from both stumps. Care being taken that the fluid should have free vent, the dress. ings were saturated externally with carbolic oil.

15th.-Patient says he is "middling well;" less heat of skin ; tongue moist; pulse 100.

17th.- Much the same. Ordered castor oil, as the bowels had not acted; also an opiate at bedtime, as he did not sleep much last night.

18th.-Expresses himself as comfortable. Bowels relieved. Pulse 100. Stumps dressed for the first time; both look well, there being no appearance of inflammation, nor yet any purulent discharge from either. Dressed with lint soaked in carbolic oil.

21st.-Going on well. No fever. Pulse 90. The thigh stump appears almost healed, the edges of the wound being entirely united, except for about an inch in the centre. The leg stump looks firm and well, except a small portion of skin (one inch) of the anterior flap, which is sloughing and discharging from beneath it a little pus.

30th.- Since last report he has made almost uninterrupted progress. From lying so constantly in one position, a small piece of skin over the sacrum threatens to slough. Ordered a water-pillow, good diet, and port wine. The thigh stump is now quite healed, except a small granulating superficial spot about the size of a shilling at the centre. In the leg stump, two-thirds of the edge of the wound are healed, the remaining portion, where the piece of skin has separated, is granulating in a healthy manner.

Feb. 14th.-Patient gets on well, and now sits up. This gives him great relief, and has allowed the skin on the sacrum to heal.

March 9th. - Both stumps healed. Patient well and strong.

In regard to the case, I should like it to be noted that1. The patient was operated on almost immediately after the accident, during the period of shock, and before decided reaction had set in.

2. All the minor arteries were secured by torsion.

3. Antiseptic dressing was used with advantage, no pus having come from the thigh stump, and very little (perhaps about an ounce) from the leg, considering a little sloughing took place in the anterior flap.

Burntisland, February, 1869 .

\section{THE MEDICAL TEACHERS' ASSOCIATION.}

A menting of this Association was held on Friday, the 18th of June, at 32A, George-street, Hanover-square, Sir W. Jenner, Bart., M.D., in the chair. After the minutes of the previous meeting had been confirmed, the Secretary announced the replies of certain of the educational bodies which are in receipt of the recent Report on Medical Education drawn up by the Association. The Secretary of the Royal College of Surgeons acknowledged the receipt of the Report in the fewest possible words, and the curtness of his answer became the subject of special comment later in the evening.

A Committee, consisting of the President and the two Secretaries, with power to add to their number, was formed to watch the question of medical education as it comes up for discussion at the forthcoming meeting of the General 